\title{
Erratum: Shock-wave structure according to a linear irreversible thermodynamic model [Phys. Rev. E 99, 023114 (2019)]
}

\author{
R. M. Velasco and F. J. Uribe (1)
}

(Received 9 December 2019; published 30 January 2020)

DOI: 10.1103/PhysRevE.101.019903

The present Erratum concerns the correction of errors we have made in the published paper. Equation (9) has a misprint on the right-hand side where it appears as $q_{x} \frac{\partial T}{\partial x}$, which must be changed to $q_{x} \frac{\partial}{\partial x}\left[\frac{1}{T}\right]$. Equation (29) has an error in the last term that is written as $a_{2} \tau^{1 / 2} \frac{d \tau}{d s}$, which must be $a_{2} \tau^{-1 / 2} \frac{d \tau}{d s}$. Consequently, Eqs. (36) are incorrect and they should be replaced with

$$
\begin{aligned}
a_{1} \frac{d v}{d s}+\frac{a_{2}}{\sqrt{\tau}} \frac{d \tau}{d s} & =\frac{\rho_{0} u_{0} \lambda}{\eta}\left(1+\tau_{0}-\frac{\tau}{v}-v\right), \\
\left(a_{1} v+a_{3} \sqrt{\tau}\right) \frac{d v}{d s}+\left(a_{4}+\frac{a_{2} v}{\sqrt{\tau}}\right) \frac{d \tau}{d s} & =\frac{\rho_{0} u_{0} \lambda}{2 \eta}\left(5 \tau_{0}+1-5 \tau-v^{2}\right) .
\end{aligned}
$$

Also, Eqs. (38) should be changed to

$$
\begin{aligned}
& \frac{d v}{d s}=\frac{\rho_{0} u_{0} \lambda}{2 \eta} \frac{\left[3 \tau-v^{2}+2 v\left(\tau_{0}+1\right)-\left(1+5 \tau_{0}\right)\right]\left(v a_{2}\right)+2\left[-v^{2}+v\left(\tau_{0}+1\right)-\tau\right]\left(\sqrt{\tau} a_{4}\right)}{\left(a_{1} a_{4}-a_{2} a_{3}\right) v \sqrt{\tau}}, \\
& \frac{d \tau}{d s}=\frac{\rho_{0} u_{0} \lambda}{2 \eta} \frac{\left[3 \tau-v^{2}+2 v\left(\tau_{0}+1\right)-\left(1+5 \tau_{0}\right)\right]\left(-v a_{1}\right)+2\left[-v^{2}+v\left(\tau_{0}+1\right)-\tau\right]\left(-\sqrt{\tau} a_{3}\right)}{\left(a_{1} a_{4}-a_{2} a_{3}\right) v} .
\end{aligned}
$$

Such an unfortunate error has driven us to an incorrect calculation of the fitted parameters. As a result, with the correct equations we have found that the conclusions we have raised about LIT1 are no longer valid. It means that with the viscosity index fixed by the experimental recommended data and the $a_{2}$ coefficient as a constant, the fit to obtain a good agreement with the normalized density profiles is not possible. Instead, the option given by LIT2 can be taken without any trouble with a new

\begin{tabular}{|c|c|c|c|c|c|c|c|c|}
\hline Model & $M$ & $\sigma$ & $a_{2}$ & $\alpha^{-1}$ & $\bar{Q}_{\rho}$ & $\bar{Q}_{\rho}^{E}[20]$ & $\lambda_{A} / \delta$ & $\lambda_{A} / \delta^{E}$ \\
\hline NSF & 1.55 & 1.6 & 0.0 & NA & 1.00 & 0.92 & 0.13 & 0.12 \\
\hline DSMC & 1.55 & 1.2 & NA & 0.6525 & 0.96 & 0.92 & 0.15 & 0.12 \\
\hline LIT2 & 1.55 & 1.5 & -0.25 & 1.02 & 1.02 & 0.92 & 0.14 & 0.12 \\
\hline NSF & 2.05 & 1.3 & 0.0 & NA & 1.08 & 0.96 & 0.20 & 0.21 \\
\hline DSMC & 2.05 & 0.81 & NA & 0.6525 & 0.99 & 0.96 & 0.20 & 0.21 \\
\hline LIT2 & 2.05 & 1.3 & -0.25 & NA & 1.07 & 0.96 & 0.20 & 0.20 \\
\hline NSF & 3.38 & 1.05 & 0.0 & NA & 1.18 & 1.02 & 0.28 & 0.28 \\
\hline DSMC & 3.38 & 0.75 & NA & 0.6525 & 1.10 & 1.02 & 0.28 & 0.28 \\
\hline LIT2 & 3.38 & 1.05 & -0.25 & NA & 1.18 & 1.02 & 0.28 & 0.28 \\
\hline NSF & 6.5 & 0.95 & 0.0 & NA & 1.24 & 1.12 & 0.25 & 0.25 \\
\hline DSMC & 6.5 & 0.72 & NA & 0.6015 & 1.13 & 1.12 & 0.26 & 0.25 \\
\hline LIT2 & 6.5 & 0.95 & -0.25 & NA & 1.23 & 1.12 & 0.25 & 0.25 \\
\hline NSF & 8.0 & 0.9 & 0.0 & NA & 1.28 & 1.13 & 0.26 & 0.24 \\
\hline DSMC & 8.0 & 0.68 & NA & 0.6015 & 1.14 & 1.13 & 0.26 & 0.24 \\
\hline LIT2 & 8.0 & 0.9 & -0.25 & NA & 1.25 & 1.13 & 0.25 & 0.24 \\
\hline NSF & 9.0 & 0.9 & 0.0 & NA & 1.26 & 1.14 & 0.24 & 0.23 \\
\hline DSMC & 9.0 & 0.72 & NA & 0.6015 & 1.15 & 1.14 & 0.23 & 0.23 \\
\hline LIT2 & 9.0 & 0.9 & -0.25 & NA & 1.25 & 1.14 & 0.24 & 0.23 \\
\hline
\end{tabular}
set of parameters, which are now shown in the corrected Table I.

TABLE I. Fitted parameters $\left(\sigma, a_{2}\right)$ in the LIT2 approach and the results for the asymmetry factor and the reciprocal width for several Mach numbers. The quantities with a superscript $E$ come from experimental data of Ref. [20]. 
The comparison between the experimental normalized density profile and the calculations done with LIT2 are essentially the same as the ones given in the original paper, at least as far as the graph resolution allows for the comparison. However, the comparisons for the temperature are not as good as the ones given with the incorrect differential equations, but provide a noticeable improvement over the Navier-Stokes-Fourier constitutive equations, especially for Mach numbers greater than 2. The whole set of tables and figures generated with the correct differential equations provided here are available on request to the interested reader.

Notice that in the present Table I we have changed the previous value of $\sigma$ for $M=6.5$ to the NSF equations and the previous value of the Mach number $(M=2.00)$, for one DSMC computation, to $M=2.05$. 